\title{
General Decay of Solutions in One-Dimensional Porous-Elastic with Memory and Distributed Delay Term
}

\author{
Abdelbaki Choucha, Djamel Ouchenane and Khaled Zennir
}

\begin{abstract}
As a continuity to the study by T. A. Apalara in [3], we consider a one-dimensional porous-elastic system with the presence of both memory and distributed delay terms in the second equation. Using the well known energy method combined with Lyapunov functionals approach, we prove a general decay result given in Theorem 2.1.
\end{abstract}

\section{Introduction}

Researchers from various fields were interested in elasticity problems, and they have been mainely attracted by the qualitative studies of different type of this problems and many results can be found in the literature. In the one-dimensional case, for instance, the combination of the elastic equations with thermal consequences causes a negative exponential to control the decay of solutions. The one-dimensional porous-elastic model is given by

$$
\begin{aligned}
\rho_{0} u_{t t} & =\mu u_{x x}+\beta \varphi_{x}, \text { in }(0, l) \times(0, L), \\
\rho_{0} k \varphi_{t t} & =\alpha \varphi_{t t}-\beta u_{x}-\tau \varphi_{t}-\xi \varphi, \text { in }(0, l) \times(0, L),
\end{aligned}
$$

and it has been studied by many authors. The first contribution in this direction was obtained by [21]. To be more precise, which was developed in [14], the authors showed that the classical elasticity theory to porous media by introducing the concept of a continuum theory of granular materials with interstitial voids into the theory of elastic solids with voids. In addition to the usual elastic effects, the materials with voids possess a microstructure with the property that the mass at each point is obtained as the product of the mass density of the material matrix by the volume fraction. This concept was introduced in the pioneered work in [17], when the authors have advanced nonlinear theory of elastic materials with voids (See [9], [10]). The basic evolution equations for one-dimensional theories of porous materials with memory effect are given by

$$
\rho u_{t t}=T_{x}, J \phi_{t t}=H_{x}+G
$$

2010 Mathematics Subject Classification. 35B40, 35L70, 93D15, 93D20.

Key words and phrases. Porous system; General decay; Exponential Decay; Memory term; Distributed delay term; Relaxation function.

Corresponding author: Abdelbaki Choucha. 
where $T$ is the stress tensor, $H$ is the equilibrated stress vector and $G$ is the equilibrated body force. The variables $u$ and $\phi$ are the displacement of the solid elastic material and the volume fraction, respectively. The constitutive equations are

$$
T=\mu u_{x}+b \phi, H=\delta \phi_{x}-\int_{0}^{t} g(t-s) \phi_{x}(s) d s, G=-b u_{x}-\xi \phi .
$$

In [21] substituting (1.2) into (1.1) is concerned

$$
\left\{\begin{array}{l}
\rho u_{t t}-\mu u_{x x}-b \phi_{x}=0, \text { in }(0,1) \times(0, \infty), \\
J \phi_{t t}-\delta \phi_{x x}+b u_{x}+\xi \phi+\int_{0}^{t} g(t-s) \phi_{x x}(x, s) d s=0, \text { in }(0,1) \times(0, \infty) .
\end{array}\right.
$$

A porous-elastic system with memory term and Neumann-Dirichlet boundary conditions where $g$ is the relaxation function it has been proved a general decay result, for more detail (see [3]). Quintanilla in [21] considered

$$
\left\{\begin{array}{l}
\rho u_{t t}=\mu u_{x x}+b \phi_{x}, x \in(0, L), t>0 \\
J \phi_{t t}=\delta \phi_{x x}-b u_{x}-\xi \phi-\tau \phi_{t}, x \in(0, L), t>0,
\end{array}\right.
$$

with initial and mixed boundary conditions and supposed that the damping in the porous equation $\left(-\tau \phi_{t}\right)$ is not enough to obtain an exponential decay but only a slow decay can be obtained. To improve this decay several other damping mechanisms were considered. In [7] Casas and Quintanilla have considered

$$
\left\{\begin{array}{l}
\rho u_{t t}=\mu u_{x x}+b \phi_{x}-\beta \theta_{x}, x \in(0, L), t>0 \\
J \phi_{t t}=\delta \phi_{x x}-b u_{x}-\xi \phi+m \theta-\tau \phi_{t}, x \in(0, L), t>0 \\
c \theta_{t}=k \theta_{x x}-\beta u_{x t}-m \phi_{t}, x \in(0, L), t>0
\end{array}\right.
$$

where $\theta$ is the temperature difference with initial and Dirichlet-Neumann boundary conditions. The authors applied the semigroup theory and the method proposed and developed in [27] to establish the exponential decay of the solutions. Later, with $\tau=0$, in [8] the same authors have proposed that the heat effect alone is not strong sufficient to bring an exponential decay but only a slow decay could be established. However, the heat effect together with micro-temperature created an exponential decay result. Similarly, when $\tau=0$ and $\gamma u_{x x t}$ is added to the first equation in (1.1), in [19], the authors have proved the lack of exponential stability. However, by taking some regular initial data a polynomial stability is obtained. Also, for $\tau=0$, problem (1.1) was considered in [25] with the following boundary conditions

$$
\left\{\begin{array}{l}
u(0, t)=\phi(0, t)=\theta(0, t)=\theta(L, t)=0, t \geq 0 \\
u(L, t)=-\int_{0}^{t} g_{1}(t-s)\left[\mu u_{x}(L, s)+b \phi(L, s)\right] d s, t \geq 0 \\
\phi(L, t)=-\delta \int_{0}^{t} g_{2}(t-s) \phi_{x}(L, s) d s, t \geq 0
\end{array}\right.
$$


where $g_{1}$ and $g_{2}$ are positive decreasing functions. They obtained a general decay result in which the usual exponential and polynomial decay rates are just special cases. ([15], [23], [20], [24], [25] and the references therein).

The viscoelastic damping is represented by a memory term in the form of a convolution which arises in the constitutive equation between the stress $\sigma(x, t)$ and the strain $\epsilon(x, t)$ (See [12], [3])

$$
\sigma(x, t)=\epsilon(x, t)+\int_{0}^{t} g(t-s) \epsilon(x, s) d s .
$$

This type of viscoelastic dissipation could be said to coincide to viscosity with null initial history because it is assumed that the strains have been zero for $-\infty<t<0$ or, equivalently, if any past strains have occurred sufficiently long ago that the effect is trivial. In other words, there will be a time prior to which all the strains which have previously occurred will have a trivial contribution. Thus, an experiment generally starts at some time $(t=0)$ when the material is free of stresses.

We must mention the pioneer works recently published by [4], the author considered a onedimensional porous thermo-elastic system with memory effects and proved a general decay result, for which exponential and polynomial decay results are special cases, depending only on the kernel of the memory effects. The obtained result were established irrespective of the wave speeds of the system (See [2], [5]). In [13] the authors investigated a porous thermo-elastic system where the heat conduction is given by Cattaneo's law and where the energy associated with the solution is not necessary positive. They introduced a stability number and proved an exponential and polynomial decay results.

Our purpose in this paper is to give a general decay result of solutions in one dimensional porouselastic system with memory and distributed delay term, our result is new and improves previous results in the literature.

Let $\mathcal{H}=(0,1) \times\left(\tau_{1}, \tau_{2}\right) \times(0, \infty)$, in the present paper, we are interested in the following problem

$$
\left\{\begin{array}{l}
\rho u_{t t}-\mu u_{x x}-b \phi_{x}=0, \\
J \phi_{t t}-\delta \phi_{x x}+b u_{x}+\xi \phi+\int_{0}^{t} g(t-s) \phi_{x x}(x, s) d s+\mu_{1} \phi_{t} \\
\int_{\tau_{1}}^{\tau_{2}}\left|\mu_{2}(s)\right| \phi_{t}(x, t-s) d s=0,
\end{array}\right.
$$

where

$$
(x, s, t) \in \mathcal{H} .
$$

As in [16], taking the following new variable

$$
z(x, \rho, s, t)=\phi_{t}(x, t-s \rho),
$$

then we obtain

$$
\left\{\begin{array}{l}
s z_{t}(x, \rho, s, t)+z_{\rho}(x, \rho, s, t)=0, \\
z(x, 0, s, t)=\phi_{t}(x, t) .
\end{array}\right.
$$


Consequently, the problem is equivalent to

$$
\left\{\begin{array}{l}
\rho u_{t t}-\mu u_{x x}-b \phi_{x}=0 \\
J \phi_{t t}-\delta \phi_{x x}+b u_{x}+\xi \phi+\int_{0}^{t} g(t-s) \phi_{x x}(x, s) d s+\mu_{1} \phi_{t} \\
\quad+\int_{\tau_{1}}^{\tau_{2}}\left|\mu_{2}(s)\right| \phi_{t}(x, t-s) d s=0, \\
s z_{t}(x, \rho, s, t)+z_{\rho}(x, \rho, s, t)=0 .
\end{array}\right.
$$

where

$$
(x, \rho, s, t) \in(0,1) \times \mathcal{H} .
$$

The system with memory and delay term acting only on the porous equation together with the initial data

$$
\left\{\begin{array}{l}
u(x, 0)=u_{0}(x), u_{t}(x, 0)=u_{1}(x), \\
\phi(x, 0)=\phi_{0}(x), \phi_{t}(x, 0)=\phi_{1}(x), x \in(0,1),
\end{array}\right.
$$

and Neumann-Dirichlet boundary conditions

$$
u_{x}(0, t)=u_{x}(1, t)=\phi(0, t)=\phi(1, t)=0, t \geq 0 .
$$

Here, $u$ is the longitudinal displacement, $\phi$ is the volume fraction of the solid elastic material and $\rho, \mu, b, J, \delta, \xi$ are positive constants with $\mu, \xi, b$ satisfying $\mu \xi>b^{2}$. The integral represents the memory and delay term with $\tau_{1}, \tau_{2}>0$ are a time delay, $\mu_{1}$ is positive constant, $\mu_{2}$ is an $L^{\infty}$ function and $g$ is the relaxation function satisfying (H1) $g \in C^{1}\left(\mathbb{R}_{+}, \mathbb{R}_{+}\right)$is a non-increasing function satisfying

$$
g(0)>0, b-\int_{0}^{\infty} g(s) d s=l>0 .
$$

(H2) There exists a positive non-increasing differentiable function $\vartheta \in\left(\mathbb{R}_{+}, \mathbb{R}_{+}\right)$satisfying

$$
g^{\prime}(t) \leq-\vartheta(t) g(t), t \geq 0
$$

(H3) $\mu_{2}:\left[\tau_{1}, \tau_{2}\right] \rightarrow \mathbb{R}$ is a bounded function satisfying

$$
\int_{\tau_{1}}^{\tau_{2}}\left|\mu_{2}(s)\right| d s \leq \mu_{1}
$$

Time delays arise in many applications because most phenomena naturally depend not only on the present state but also on some past occurrences In recent years, the control of PDEs with time delay effects has become an active area of research, see for example [26] and references therein. In many cases it was shown that delay is a source of instability unless additional condition or control terms are used, see [11] Therefore, the stability issue of systems with delay is of theoretical and practical great importance. 
It is well know that, in the single wave equation, if $\mu_{2}=0$ that is, in absence of a decay, the energy of system exponentially decays (see [4]) On the contrary, if $\mu_{1}=0$, that is, there exists only the delay part in the interior, the system becomes unstable (see [11]). It is shown that a small delay in a boundary control can turn such a well-behaved hyperbolic system into a wild one and therefore, delay becomes a source of instability. To stabilize a hyperbolic system involving input delay terms, additional control terms will be necessary (see [16], [18]).

In what follows, we consider $(u, \phi)$ to be a solution of system (1.6)-(1.8) with the regularity needed to justify the calculations in this paper. We specify Section 2 to the statements and prove of our stability result. We use $\mathrm{c}$ throughout this paper to denote a generic positive constant. Meanwhile, from (1.6) and (1.8), it follows that

$$
\frac{d^{2}}{d t^{2}} \int_{0}^{1} u(x, t) d x=0
$$

So, by solving (1.12) and using the initial data of $u$, we get

$$
\int_{0}^{1} u(x, t) d x=t \int_{0}^{1} u_{1}(x) d x+\int_{0}^{1} u_{0}(x) d x
$$

Consequently, if we let

$$
\bar{u}(x, t)=u(x, t)-t \int_{0}^{1} u_{1}(x) d x-\int_{0}^{1} u_{0}(x) d x
$$

we get

$$
\int_{0}^{1} \bar{u}(x, t) d x=0, \forall t \geq 0
$$

Therefore, the use of Poincare's inequality for $\bar{u}$ is justified. In addition, simple substitution shows that $(\bar{u}, \phi)$ satisfies system (1.6) with initial data for $\bar{u}$ given as

$$
\overline{u_{0}}(x)=u_{0}(x)-\int_{0}^{1} u_{0}(x) d x \text { and } \overline{u_{1}}(x)=u_{1}(x)-\int_{0}^{1} u_{1}(x) d x .
$$

Henceforth, we work with $\bar{u}$ instead of $u$ but write $u$ for simplicity of notation.

\section{Main Result}

In this section, we state and prove our decay result for the energy of the system (1.6)-(1.8) using the multiplier technique. We need the following lemmas. 
Lemma 2.1. The energy functional $E$, defined by

$$
\begin{aligned}
E(t)= & \frac{1}{2} \int_{0}^{1}\left[\rho u_{t}^{2}+\mu u_{x}^{2}+J \phi_{t}^{2}+\left(\delta-\int_{0}^{t} g(s) d s\right) \phi_{x}^{2}+\xi \phi^{2}+2 b u_{x} \phi\right] d x \\
& +\frac{1}{2} g \circ \phi_{x}+\frac{1}{2} \int_{0}^{1} \int_{0}^{1} \int_{\tau_{1}}^{\tau_{2}} s\left|\mu_{2}(s)\right| z^{2}(x, \rho, s, t) d s d \rho d x
\end{aligned}
$$

satisfies

$$
E^{\prime}(t)=\frac{1}{2} g^{\prime} \circ \phi_{x}-\frac{1}{2} g(t) \int_{0}^{1} \phi_{x}^{2} d x-\left(\mu_{1}-\int_{\tau_{1}}^{\tau_{2}}\left|\mu_{2}(s)\right| d s\right) \int_{0}^{1} \phi_{t}^{2} d x
$$

and

$$
E^{\prime}(t) \leq \frac{1}{2} g^{\prime} \circ \phi_{x}-\eta_{0} \int_{0}^{1} \phi_{t}^{2} d x \leq 0
$$

where $\eta_{0}=\mu_{1}-\int_{\tau_{1}}^{\tau_{2}}\left|\mu_{2}(s)\right| d s \geq 0$ and $g \circ v=\int_{0}^{1} \int_{0}^{t} g(t-s)\left(v_{x}(t)-v_{x}(s)\right)^{2} d s d x$.

Proof. Multiplying the first equation of (1.6) by $u_{t}$ and the second equation by $\phi_{t}$, then integration by parts over $(0,1)$, and using $(1.8)$, we get

$$
\begin{aligned}
& \frac{1}{2} \frac{d}{d t} \int_{0}^{1}\left[\rho u_{t}^{2}+\mu u_{x}^{2}+J \phi_{t}^{2}+\delta \phi_{x}^{2}+\xi \phi^{2}+2 b u_{x} \phi\right] d x-\int_{0}^{1} \phi_{x t} \int_{0}^{t} g(t-s) \phi_{x}(s) d s d x \\
& +\mu_{1} \int_{0}^{1} \phi_{t}^{2} d x+\int_{0}^{1} \phi_{t} \int_{\tau_{1}}^{\tau_{2}}\left|\mu_{2}(s)\right| z(x, 1, s, t) d s d x=0 .
\end{aligned}
$$

The last term in the left hand side of (2.4) is estimated as follows.

$$
\begin{aligned}
-\int_{0}^{1} \phi_{x t} & \int_{0}^{t} g(t-s) \phi_{x}(s) d s d x \\
& =\int_{0}^{1} \phi_{x t} \int_{0}^{t} g(t-s)\left(\phi_{x}(t)-\phi_{x}(s)\right) d s d x-\int_{0}^{t} g(s) d s \int_{0}^{1} \phi_{x t} \phi_{x} d x \\
& =\frac{1}{2} \frac{d}{d t} g \circ \phi_{x}-\frac{1}{2} \frac{d}{d t} \int_{0}^{t} g(s) d s \int_{0}^{1} \phi_{x}^{2} d x-\frac{1}{2} g^{\prime} \circ \phi_{x}+\frac{1}{2} g(t) \int_{0}^{1} \phi_{x}^{2} d x .
\end{aligned}
$$

Now, multiplying the last equation in (1.6) by $z\left|\mu_{2}(s)\right|$, and integrating the result over $(0,1) \times$ $(0,1) \times\left(\tau_{1}, \tau_{2}\right)$

$$
\begin{aligned}
\frac{d}{d t} & \frac{1}{2} \int_{0}^{1} \int_{0}^{1} \int_{\tau_{1}}^{\tau_{2}} s\left|\mu_{2}(s)\right| z^{2}(x, \rho, s, t) d s d \rho d x \\
& =-\int_{0}^{1} \int_{0}^{1} \int_{\tau_{1}}^{\tau_{2}}\left|\mu_{2}(s)\right| z z_{\rho}(x, \rho, s, t) d s d \rho d x \\
& =-\frac{1}{2} \int_{0}^{1} \int_{0}^{1} \int_{\tau_{1}}^{\tau_{2}}\left|\mu_{2}(s)\right| \frac{d}{d \rho} z^{2}(x, \rho, s, t) d s d \rho d x
\end{aligned}
$$




$$
\begin{aligned}
& =\frac{1}{2} \int_{0}^{1} \int_{\tau_{1}}^{\tau_{2}}\left|\mu_{2}(s)\right|\left(z^{2}(x, 0, s, t)-z^{2}(x, 1, s, t)\right) d s d x \\
& =\frac{1}{2} \int_{\tau_{1}}^{\tau_{2}}\left|\mu_{2}(s)\right| d s \int_{0}^{1} \phi_{t}^{2} d x-\frac{1}{2} \int_{0}^{1} \int_{\tau_{1}}^{\tau_{2}}\left|\mu_{2}(s)\right| z^{2}(x, 1, s, t) d s d x .
\end{aligned}
$$

Now, using Young's inequality, we have

$$
\begin{aligned}
E^{\prime}(t) & \leq \frac{1}{2} g^{\prime} \circ \phi_{x}-\frac{1}{2} g(t) \int_{0}^{1} \phi_{x}^{2} d x-\left(\mu_{1}-\int_{\tau_{1}}^{\tau_{2}}\left|\mu_{2}(s)\right| d s\right) \int_{0}^{1} \phi_{t}^{2} d x \\
& \leq \frac{1}{2} g^{\prime} \circ \phi_{x}-\left(\mu_{1}-\int_{\tau_{1}}^{\tau_{2}}\left|\mu_{2}(s)\right| d s\right) \int_{0}^{1} \phi_{t}^{2} d x
\end{aligned}
$$

then, by $(H 3)$, there exists a positive constant $\eta_{0}$ such that

$$
E^{\prime}(t) \leq \frac{1}{2} g^{\prime} \circ \phi_{x}-\eta_{0} \int_{0}^{1} \phi_{t}^{2} d x
$$

then we obtain $E$ is a non-increasing function.

Lemma 2.2. The functional

$$
D_{1}(t):=J \int_{0}^{1} \phi_{t} \phi d x+\frac{b \rho}{\mu} \int_{0}^{1} \phi \int_{0}^{x} u_{t}(y) d y d x
$$

satisfies

$$
\begin{aligned}
D_{1}^{\prime}(t) \leq & -\frac{l}{2} \int_{0}^{1} \phi_{x}^{2} d x-\mu_{3} \int_{0}^{1} \phi^{2} d x+\varepsilon_{1} \int_{0}^{1} u_{t}^{2} d x+c\left(1+\frac{1}{\varepsilon}\right) \int_{0}^{1} \phi_{t}^{2} d x \\
& +c g \circ \phi_{x}+c \int_{0}^{1} \int_{\tau_{1}}^{\tau_{2}}\left|\mu_{2}(s)\right| z^{2}(x, 1, s, t) d s d x,
\end{aligned}
$$

where $\mu_{3}=\xi-\frac{b^{2}}{\mu}>0$.

Proof. Direct computation using integration by parts and Young's inequality, for $\varepsilon_{1}>0$, yields

$$
\begin{aligned}
D_{1}^{\prime}(t)= & -\delta \int_{0}^{1} \phi_{x}^{2} d x-\left(\xi-\frac{b^{2}}{\mu}\right) \int_{0}^{1} \phi^{2} d x+J \int_{0}^{1} \phi_{t}^{2} d x+\frac{b \rho}{\mu} \int_{0}^{1} \phi_{t} \int_{0}^{x} u_{t}(y) d y d x \\
& +\int_{0}^{1} \phi_{x} \int_{0}^{t} g(t-s) \phi_{x}(s) d s d x-\mu_{1} \int_{0}^{1} \phi_{t} \phi d x-\int_{0}^{1} \phi \int_{\tau_{1}}^{\tau_{2}}\left|\mu_{2}(s)\right| z(x, 1, s, t) d s d x \\
\leq & -\delta \int_{0}^{1} \phi_{x}^{2} d x-\left(\xi-\frac{b^{2}}{\mu}\right) \int_{0}^{1} \phi^{2} d x+c\left(1+\frac{1}{\varepsilon_{1}}\right) \int_{0}^{1} \phi_{t}^{2} d x+\varepsilon_{1} \int_{0}^{1}\left(\int_{0}^{x} u_{t}(y) d y\right)^{2} d x \\
& +\int_{0}^{1} \phi_{x} \int_{0}^{t} g(t-s) \phi_{x}(s) d s d x-\mu_{1} \int_{0}^{1} \phi_{t} \phi d x-\int_{0}^{\tau_{2}}\left|\int_{\tau_{1}}(s)\right| z(x, 1, s, t) d s d x .
\end{aligned}
$$


By Cauchy-Schwartz inequality, it is clear that

$$
\int_{0}^{1}\left(\int_{0}^{x} u_{t}(y) d y\right)^{2} d x \leq \int_{0}^{1}\left(\int_{0}^{1} u_{t} d x\right)^{2} d x \leq \int_{0}^{1} u_{t}^{2} d x .
$$

So, estimate (2.10) becomes

$$
\begin{aligned}
D_{1}^{\prime}(t) \leq & -\delta \int_{0}^{1} \phi_{x}^{2} d x-\left(\xi-\frac{b^{2}}{\mu}\right) \int_{0}^{1} \phi^{2} d x+c\left(1+\frac{1}{\varepsilon_{1}}\right) \int_{0}^{1} \phi_{t}^{2} d x+\varepsilon_{1} \int_{0}^{1} u_{t}^{2} d x \\
& -\mu_{1} \int_{0}^{1} \phi_{t} \phi d x-\int_{0}^{1} \phi \int_{\tau_{1}}^{\tau_{2}}\left|\mu_{2}(s)\right| z(x, 1, s, t) d s d x \\
& +\int_{0}^{1} \phi_{x} \int_{0}^{t} g(t-s) \phi_{x}(s) d s d x
\end{aligned}
$$

The last term in the RHS of (2.11) is estimated as follows:

$$
\begin{aligned}
& \int_{0}^{1} \phi_{x} \int_{0}^{t} g(t-s) \phi_{x}(s) d s d x \\
& =\int_{0}^{t} g(s) d s \int_{0}^{1} \phi_{x}^{2} d x-\int_{0}^{1} \phi_{x} \int_{0}^{t} g(t-s)\left(\phi_{x}(t)-\phi_{x}(s)\right) d s d x \\
& \leq\left(\delta_{1}+\int_{0}^{t} g(s) d s\right) \int_{0}^{1} \phi_{x}^{2} d x+\frac{1}{4 \delta_{1}}\left(\int_{0}^{t} g(s) d s\right) g \circ \phi_{x},
\end{aligned}
$$

where we have used Cauchy-Schwartz, Young's and poincare's inequalities, for $\delta_{1}, \varepsilon_{2}, \varepsilon_{3}>0$. By substituting (2.12) into(2.10), we obtain

$$
\begin{aligned}
D_{1}^{\prime}(t) \leq & -\left(\delta-\int_{0}^{t} g(s) d s-\delta_{1}-\mu_{1} c \delta_{2}-\mu_{1} c \delta_{3}\right) \int_{0}^{1} \phi_{x}^{2} d x-\left(\xi-\frac{b^{2}}{\mu}\right) \int_{0}^{1} \phi^{2} d x \\
& +\varepsilon_{1} \int_{0}^{1} u_{t}^{2} d x+\left(c\left(1+\frac{1}{\varepsilon_{1}}\right)+\frac{\mu_{1}}{4 \delta_{2}}\right) \int_{0}^{1} \phi_{t}^{2} d x+\frac{1}{4 \delta_{1}}\left(\int_{0}^{t} g(s) d s\right) g \circ \phi_{x} \\
& +\frac{1}{4 \delta_{3}} \int_{0}^{t} \int_{\tau_{1}}^{\tau_{2}}\left|\mu_{2}(s)\right| z^{2}(x, 1, s, t) d s d x
\end{aligned}
$$

Bearing in mind that $\mu \xi>b^{2}$ and using the fact that $\delta-\int_{0}^{t} g(s) d s \geq l$, and letting $\delta_{1}=\frac{l}{6}, \delta_{2}=\delta_{3}=\frac{l}{6 c \mu_{1}}$, we obtain estimate (2.9).

In the following Lemma, we use the essential hypothesis that the wave speeds of the system are equal

$$
\frac{\mu}{\rho}=\frac{\delta}{J}
$$

Lemma 2.3. Assume that (H1) and (2.14) hold. Then the functional

$$
D_{2}(t):=\int_{0}^{1} \phi_{x} u_{t} d x+\int_{0}^{1} \phi_{t} u_{x} d x-\frac{\rho}{\mu J} \int_{0}^{1} u_{t} \int_{0}^{t} g(t-s) \phi_{x}(s) d s d x
$$


satisfies, for any $\varepsilon_{2}>0$

$$
\begin{aligned}
D_{2}^{\prime}(t) \leq & -\frac{b}{2 J} \int_{0}^{1} u_{x}^{2} d x+c\left(1+\frac{1}{\varepsilon_{2}}\right) \int_{0}^{1} \phi_{x}^{2} d x+c \varepsilon_{2} \int_{0}^{1} u_{t}^{2} d x \\
& +c \int_{0}^{1} \phi_{t}^{2}+c g \circ \phi_{x}-\frac{c}{\varepsilon_{2}} g^{\prime} \circ \phi_{x}+c \mu_{1} \int_{0}^{1} \int_{\tau_{1}}^{\tau_{2}}\left|\mu_{2}(s)\right| z^{2}(x, 1, s, t) d x .
\end{aligned}
$$

Proof. By differentiating $D_{2}$, then using (1.6), integration by parts, and (1.8) we obtain

$$
\begin{aligned}
D_{2}^{\prime}(t)= & -\frac{b}{J} \int_{0}^{1} u_{x}^{2} d x+\left(\frac{\delta}{J}-\frac{\mu}{\rho}\right) \int_{0}^{1} u_{x} \phi_{x x} d x+\frac{b}{\rho} \int_{0}^{1} \phi_{x}^{2} d x-\frac{\rho g(0)}{\mu J} \int_{0}^{1} u_{t} \phi_{x} d x \\
& -\frac{\xi}{J} \int_{0}^{1} u_{x} \phi d x-\frac{b}{\mu J} \int_{0}^{1} \phi_{x} \int_{0}^{t} g(t-s) \phi_{x}(s) d s d x \\
& -\frac{\rho}{\mu J} \int_{0}^{1} u_{t} \int_{0}^{t} g^{\prime}(t-s) \phi_{x}(s) d s d x \\
& -\frac{\mu_{1}}{J} \int_{0}^{1} \phi_{t} u_{x} d x-\frac{1}{J} \int_{0}^{1} u_{x} \int_{\tau_{1}}^{\tau_{2}}\left|\mu_{2}(s)\right| z^{2}(x, 1, s, t) d s d x .
\end{aligned}
$$

In what follows, we estimate the last five terms in the right hand side of (2.16), using Young's, Cauchy-Schwartz, and Poincare's inequalities. For $\delta_{4}, \delta_{5}, \varepsilon_{2}>0$, we have

$$
-\frac{\xi}{J} \int_{0}^{1} u_{x} \phi d x \leq \frac{\xi}{J} \delta_{4} \int_{0}^{1} u_{x}^{2} d x+\frac{\xi}{4 J \delta_{4}} \int_{0}^{1} \phi^{2} d x .
$$

By letting $\delta_{4}=\frac{b}{6 \xi}$, using Poincar's inequality, we get

$$
\begin{gathered}
-\frac{\xi}{J} \int_{0}^{1} u_{x} \phi d x \leq \frac{b}{6 J} \int_{0}^{1} u_{x}^{2} d x+c \int_{0}^{1} \phi^{2} d x \\
-\frac{b}{\mu J} \int_{0}^{1} \phi_{x} \int_{0}^{t} g(t-s) \phi_{x}(s) d s d x \\
=\frac{b}{\mu J} \int_{0}^{1} \phi_{x} \int_{0}^{t} g(t-s)\left(\phi_{x}(t)-\phi_{x}(s)\right) d s d x-\frac{b}{\mu J} \int_{0}^{t} g(s) d s \int_{0}^{1} \phi_{x}^{2} d x \\
\leq\left(\delta_{5}-\frac{b}{\mu J}\right) \int_{0}^{t} g(s) d s \int_{0}^{1} \phi_{x}^{2} d x+\frac{c}{\delta_{5}} g \circ \phi_{x} .
\end{gathered}
$$

By letting $\delta_{5}=\frac{b}{\mu J}$ we get

$$
\begin{array}{r}
-\frac{b}{\mu J} \int_{0}^{1} \phi_{x} \int_{0}^{t} g(t-s) \phi_{x}(s) d s d x \leq c g \circ \phi_{x} \\
-\frac{\rho}{\mu J} \int_{0}^{1} u_{t} \int_{0}^{t} g^{\prime}(t-s) \phi_{x}(s) d s d x
\end{array}
$$




$$
\begin{aligned}
& =\frac{b}{\mu J} \int_{0}^{1} u_{t} \int_{0}^{t} g^{\prime}(t-s)\left(\phi_{x}(t)-\phi_{x}(s)\right) d s d x-\frac{b}{\mu J} \int_{0}^{t} g^{\prime}(s) d s \int_{0}^{1} u_{t} \phi_{x} d x \\
& \leq \frac{\rho \varepsilon_{2}}{2 \mu J} \int_{0}^{1} u_{t}^{2} d x+\frac{\rho g(0)}{\mu J} \int_{0}^{1} u_{t} \phi_{x} d x-\frac{\rho g(t)}{\mu J} \int_{0}^{1} u_{t} \phi_{x} d x \\
& +\frac{\rho}{2 \mu J \varepsilon_{2}} \int_{0}^{1} g^{\prime}(s) d s \int_{0}^{1} \int_{0}^{t} g^{\prime}(t-s)\left(\phi_{x}(t)-\phi_{x}(s)\right)^{2} d s d x \\
& \leq \frac{\rho \varepsilon_{2}}{\mu J} \int_{0}^{1} u_{t}^{2} d x+\frac{\rho}{2 \mu J \varepsilon_{2}}\left(\int_{0}^{1} g^{\prime}(s) d s\right) g^{\prime} \circ \phi_{x}+\frac{\rho g(0)}{\mu J} \int_{0}^{1} u_{t} \phi_{x} d x \\
& +\frac{\rho g(t)}{2 \mu J \varepsilon_{2}} \int_{0}^{1} u_{t} \phi_{x} d x \\
& \leq \frac{\rho \varepsilon_{2}}{\mu J} \int_{0}^{1} u_{t}^{2} d x+\frac{\rho}{2 \mu J \varepsilon_{2}}\left(\int_{0}^{1} g^{\prime}(s) d s\right) g^{\prime} \circ \phi_{x}+\frac{\rho g(0)}{\mu J} \int_{0}^{1} u_{t} \phi_{x} d x \\
& +\frac{\rho(g(t))^{2}}{2 \mu J \varepsilon_{2}} \int_{0}^{1} \phi_{x}^{2} d x \\
& \leq c \varepsilon_{2} \int_{0}^{1} u_{t}^{2} d x+\frac{c}{\varepsilon_{2}} \int_{0}^{1} \phi_{x}^{2} d x+\frac{\rho g(0)}{\mu J} \int_{0}^{1} u_{t} \phi_{x} d x-\frac{c}{\varepsilon_{2}} g^{\prime} \circ \phi_{x}, \\
& -\frac{\mu_{1}}{J} \int_{0}^{1} \phi_{t} u_{x} d x \leq \frac{\mu_{1} \delta_{6}}{2 J} \int_{0}^{1} \phi_{t}^{2} d x+\frac{\mu_{1}}{2 J \delta_{6}} \int_{0}^{1} u_{x}^{2} d x \\
& \frac{1}{J} \int_{0}^{1} u_{x} \int_{\tau_{1}}^{\tau_{2}}\left|\mu_{2}(s)\right| z(x, 1, s, t) d s d x \\
& \leq \frac{\delta_{7} \mu_{1}}{2 J} \int_{0}^{1} u_{x}^{2} d x+\frac{1}{2 J \delta_{7}} \int_{0}^{1} \int_{\tau_{1}}^{\tau_{2}}\left|\mu_{2}(s)\right| z^{2}(x, 1, s, t) d s .
\end{aligned}
$$

The replacement of (2.17)-(2.21) into (2.16), and by letting $\delta_{6}=\delta_{7}=\frac{b}{4 \mu_{1}}$, bearing in the mind (2.14), yields (2.15).

Lemma 2.4. The functional

$$
D_{3}(t):=-\rho \int_{0}^{1} u_{t} u d x
$$

satisfies

$$
D_{3}^{\prime}(t) \leq-\rho \int_{0}^{1} u_{t}^{2} d x+\frac{3 \mu}{2} \int_{0}^{1} u_{x}^{2} d x+c \int_{0}^{1} \phi_{x}^{2} d x
$$

Proof. Direct computations give

$$
D_{3}^{\prime}(t)=-\rho \int_{0}^{1} u_{t}^{2} d x+\mu \int_{0}^{1} u_{x}^{2} d x+b \int_{0}^{1} u_{x} \phi d x .
$$


Estimat (2.22) easily follows by using Young's and Poincaré inequalities.

$$
\begin{aligned}
D_{3}^{\prime}(t) & \leq-\rho \int_{0}^{1} u_{t}^{2} d x+\mu \int_{0}^{1} u_{x}^{2} d x+b \varepsilon \int_{0}^{1} u_{x}^{2} d x+\frac{b}{4 \varepsilon} \int_{0}^{1} \phi^{2} d x \\
& \leq-\rho \int_{0}^{1} u_{t}^{2} d x+\mu \int_{0}^{1} u_{x}^{2} d x+b \varepsilon \int_{0}^{1} u_{x}^{2} d x+\frac{b c}{4 \varepsilon} \int_{0}^{1} \phi_{x}^{2} d x
\end{aligned}
$$

by letting $\varepsilon=\frac{\mu}{2 b}$, we obtain (2.22).

Now, let us introduce the following functional used by

Lemma 2.5. The functional

$$
D_{4}(t):=\int_{0}^{1} \int_{0}^{1} \int_{\tau_{1}}^{\tau_{2}} s e^{-s \rho}\left|\mu_{2}(s)\right| z^{2}(x, \rho, s, t) d s d \rho d x
$$

satisfies,

$$
\begin{aligned}
D_{4}^{\prime}(t) \leq & -\eta_{1} \int_{0}^{1} \int_{0}^{1} \int_{\tau_{1}}^{\tau_{2}} s\left|\mu_{2}(s)\right| z^{2}(x, \rho, s, t) d s d \rho d x+\mu_{1} \int_{0}^{1} \phi_{t}^{2} d x \\
& -\eta_{1} \int_{0}^{1} \int_{\tau_{1}}^{\tau_{2}}\left|\mu_{2}(s)\right| z^{2}(x, 1, s, t) d s d x
\end{aligned}
$$

where $\eta_{1}$ is a positive constant.

Proof. By differentiating $D_{4}$, with respect to $t$ and using the last equation in $(H 3)$, we have

$$
\begin{aligned}
D_{4}^{\prime}(t)= & -2 \int_{0}^{1} \int_{0}^{1} \int_{\tau_{1}}^{\tau_{2}} e^{-s \rho}\left|\mu_{2}(s)\right| z z_{\rho}(x, \rho, s, t) d s d \rho d x \\
= & -\int_{0}^{1} \int_{0}^{1} \int_{\tau_{1}}^{\tau_{2}} s e^{-s \rho}\left|\mu_{2}(s)\right| z^{2}(x, \rho, s, t) d s d \rho d x \\
& -\int_{0}^{1} \int_{\tau_{1}}^{\tau_{2}}\left|\mu_{2}(s)\right|\left[e^{-s} z^{2}(x, 1, s, t)-z^{2}(x, 0, s, t)\right] d s d x .
\end{aligned}
$$

Using the fact that $z(x, 0, s, t)=\phi_{t}(x, t)$, and $e^{-s} \leq e^{-s \rho} \leq 1$, for all $0<\rho<1$, we obtain

$$
\begin{aligned}
D_{4}^{\prime}(t)= & -\eta_{1} \int_{0}^{1} \int_{0}^{1} \int_{\tau_{1}}^{\tau_{2}} s\left|\mu_{2}(s)\right| z^{2}(x, \rho, s, t) d s d \rho d x \\
& -\int_{0}^{1} \int_{\tau_{1}}^{\tau_{2}} e^{-s}\left|\mu_{2}(s)\right| z^{2}(x, 1, s, t) d s d x+\int_{\tau_{1}}^{\tau_{2}}\left|\mu_{2}(s)\right| d s \int_{0}^{1} \phi_{t}^{2} d x .
\end{aligned}
$$


Because $-e^{-s}$ is a increasing function, we have $-e^{-s} \leq-e^{-\tau_{2}}$, for all $s \in\left[\tau_{1}, \tau_{2}\right]$. Finally, setting $\eta_{1}=e^{-\tau_{2}}$ and recalling (H3), we obtain (2.23). We are now ready to prove the main result.

Theorem 2.1. Assume (H1), (H2), (H3) and (2.14) hold. Then, for any $t_{0}>0$, there exist positive constants $\alpha$ and $\beta$ such that the energy functional given by (2.1) satisfies

$$
E(t) \leq \alpha e^{-\beta \int_{t_{0}}^{t} \vartheta(s) d s}, \forall t \geq t_{0} .
$$

Proof. We define a Lyapunov functional

$$
\mathcal{L}(t):=N E(t)+N_{1} D_{1}(t)+N_{2} D_{2}(t)+D_{3}(t)+N_{4} D_{4}(t),
$$

where $N, N_{1}, N_{2}$, and $N_{4}$ are positive constants to be selected later.

By differentiating (2.25) and using (2.1), (2.9), (2.15), (2.22), (2.23), we have

$$
\begin{aligned}
\mathcal{L}^{\prime}(t) \leq & -\left[\frac{l N_{1}}{2}-c N_{2}\left(1+\frac{1}{\varepsilon_{2}}\right)-c\right] \int_{0}^{1} \phi_{x}^{2} d x-\left[\rho-N_{1} \varepsilon_{1}-N_{2} c \varepsilon_{2}\right] \int_{0}^{1} u_{t}^{2} d x \\
& -\left[\frac{b N_{2}}{2 J}-\frac{3 \mu}{2}\right] \int_{0}^{1} u_{x}^{2} d x-\left[\eta_{0} N-c N_{1}\left(1+\frac{1}{\varepsilon_{1}}\right)-N_{2} c-\mu_{1} N_{4}\right] \int_{0}^{1} \phi_{t}^{2} d x \\
& -N_{1} \mu_{3} \int_{0}^{1} \phi^{2} d x-\left[N_{4} \eta_{1}-c N_{1}-c N_{2}\right] \int_{0}^{1} \int_{\tau_{1}}^{\tau_{2}}\left|\mu_{2}(s)\right| z^{2}(x, 1, s, t) d s d x \\
& +c\left[N_{1}+N_{2}\right] g \circ \phi_{x}+\left[\frac{N}{2}-\frac{c N_{2}}{\varepsilon_{2}}\right] g^{\prime} \circ \phi_{x} \\
& -N_{4} \eta_{1} \int_{0}^{1} \int_{0}^{1} \int_{\tau_{1}}^{\tau_{2}} s\left|\mu_{2}(s)\right| z^{2}(x, \rho, s, t) d s d \rho d x .
\end{aligned}
$$

By setting

$$
\varepsilon_{1}=\frac{\rho}{4 N_{1}}, \varepsilon_{2}=\frac{\rho}{4 c N_{2}}
$$

we obtain

$$
\begin{aligned}
\mathcal{L}^{\prime}(t) \leq & -\left[\frac{l N_{1}}{2}-c N_{2}\left(1+N_{2}\right)-c\right] \int_{0}^{1} \phi_{x}^{2} d x-\frac{\rho}{2} \int_{0}^{1} u_{t}^{2} d x \\
& -\left[\frac{b N_{2}}{2 J}-\frac{3 \mu}{2}\right] \int_{0}^{1} u_{x}^{2} d x-\left[\eta_{0} N-c N_{1}\left(1+N_{1}\right)-c N_{2}-\mu_{1} N_{4}\right] \int_{0}^{1} \phi_{t}^{2} d x \\
& -N_{1} \mu_{3} \int_{0}^{1} \phi^{2} d x-\left[N_{4} \eta_{1}-c N_{1}-c N_{2}\right] \int_{0}^{1} \int_{\tau_{1}}^{\tau_{2}}\left|\mu_{2}(s)\right| z^{2}(x, 1, s, t) d s d x \\
& +c\left[N_{1}+N_{2}\right] g \circ \phi_{x}+\left[\frac{N}{2}-c N_{2}^{2}\right] g^{\prime} \circ \phi_{x} \\
& -N_{4} \eta_{1} \int_{0}^{1} \int_{0}^{1} \int_{\tau_{1}}^{\tau_{2}} s\left|\mu_{2}(s)\right| z^{2}(x, \rho, s, t) d s d \rho d x .
\end{aligned}
$$


Next, we carefully choose our constants so that the terms inside the brackets are positive. We choose $N_{2}$ large enough such that

$$
\alpha_{1}=\frac{b N_{2}}{2 J}-\frac{3 \mu}{2}>0,
$$

then We choose $N_{1}$ large enough such that

$$
\alpha_{2}=\frac{l N_{1}}{4}-c N_{2}\left(1+N_{2}\right)-c>0
$$

then We choose $N_{4}$ large enough such that

$$
\alpha_{3}=N_{4} \eta_{1}-c N_{1}-c N_{2}>0
$$

thus, we arrive at

$$
\begin{aligned}
\mathcal{L}^{\prime}(t) \leq & -\alpha_{2} \int_{0}^{1} \phi_{x}^{2} d x-\alpha_{0} \int_{0}^{1} \phi^{2} d x-\frac{\rho}{2} \int_{0}^{1} u_{t}^{2} d x-\alpha_{1} \int_{0}^{1} u_{x}^{2} d x-\left[\eta_{0} N-c\right] \int_{0}^{1} \phi_{t}^{2} d x \\
& +\left[\frac{N}{2}-c\right] g^{\prime} \circ \phi_{x}+c g \circ \phi_{x}-\alpha_{3} \int_{0}^{1} \int_{\tau_{1}}^{\tau_{2}}\left|\mu_{2}(s)\right| z^{2}(x, 1, s, t) d s d x \\
& -\alpha_{4} \int_{0}^{1} \int_{0}^{1} \int_{\tau_{1}}^{\tau_{2}} s\left|\mu_{2}(s)\right| z^{2}(x, \rho, s, t) d s d \rho d x
\end{aligned}
$$

where $\alpha_{0}=\mu_{3} N_{1}=\left(\xi-\frac{b^{2}}{\mu}\right) N_{1}$. On the other hand, if we let

$$
\mathfrak{L}(t)=N_{1} D_{1}(t)+N_{2} D_{2}(t)+D_{3}(t)+N_{4} D_{4}(t),
$$

then

$$
\begin{aligned}
|\mathfrak{L}(t)| \leq & J N_{1} \int_{0}^{1}\left|\phi \phi_{t}\right| d x+N_{2} \int_{0}^{1}\left|\phi_{x} u_{t}+u_{x} \phi_{t}-\frac{\rho}{\mu J} u_{t} \int_{0}^{t} g(t-s) \phi_{x}(s) d s\right| d x \\
& \frac{b \rho N_{1}}{\mu} \int_{0}^{1}\left|\phi \int_{0}^{x} u_{t}(y) d y\right| d x+\rho \int_{0}^{1}\left|u_{t} u\right| d x \\
& +N_{4} \int_{0}^{1} \int_{0}^{1} \int_{\tau_{1}}^{\tau_{2}} s e^{-s \rho}\left|\mu_{2}(s)\right| z^{2}(x, \rho, s, t) d s d \rho d x .
\end{aligned}
$$

Exploiting Young's, Cauchy-Schwartz, and Poincaré inequalities, we obtain

$$
\begin{aligned}
|\mathfrak{L}(t)| & \leq c \int_{0}^{1}\left(u_{t}^{2}+\phi_{t}^{2}+\phi_{x}^{2}+u_{x}^{2}+\phi^{2}\right) d x+c g \circ \phi_{x} \\
& +c \int_{0}^{1} \int_{0}^{1} \int_{\tau_{1}}^{\tau_{2}} s\left|\mu_{2}(s)\right| z^{2}(x, \rho, s, t) d s d \rho \\
& \leq c E(t) .
\end{aligned}
$$


Consequently, we obtain

$$
|\mathfrak{L}(t)|=|\mathcal{L}(t)-N E(t)| \leq c E(t),
$$

that is

$$
(N-c) E(t) \leq \mathcal{L}(t) \leq(N+c) E(t) .
$$

Now, by choosing $N$ large enough such that

$$
\frac{N}{2}-c>0, N-c>0, N \eta_{0}-c>0
$$

and exploiting (2.1), estimates (2.26) and (2.27), respectively, give

$$
\mathcal{L}^{\prime}(t) \leq-k_{1} E(t)+k_{2} g \circ \phi_{x}, \forall t \geq t_{0},
$$

and

$$
c_{2} E(t) \leq \mathcal{L}(t) \leq c_{3} E(t), \forall t \geq 0
$$

for some $k_{1}, k_{2}, c_{2}, c_{3}>0$.

By multiplying (2.28) by $\vartheta(t)$, we obtain

$$
\vartheta(t) \mathcal{L}^{\prime}(t) \leq-k_{1} \vartheta(t) E(t)+k_{2} \vartheta(t) g \circ \phi_{x}, \forall t \geq t_{0} .
$$

The final term in (2.30) is estimated as following, using (1.10), we have

$$
\begin{aligned}
\vartheta(t) g \circ \phi_{x} & =\vartheta(t) \int_{0}^{1} \int_{0}^{t} g(t-s)\left(\phi_{x}(t)-\phi_{x}(s)\right)^{2} d s d x \\
& \leq \int_{0}^{1} \int_{0}^{t} \vartheta(t-s) g(t-s)\left(\phi_{x}(t)-\phi_{x}(s)\right)^{2} d s d x \\
& \leq-\int_{0}^{1} \int_{0}^{t} g^{\prime}(t-s)\left(\phi_{x}(t)-\phi_{x}(s)\right)^{2} d s d x=-g^{\prime} \circ \phi_{x} \\
& \leq-2 E^{\prime}(t) .
\end{aligned}
$$

Thus, (2.30) becomes

$$
\vartheta(t) \mathcal{L}^{\prime}(t) \leq-k_{1} \vartheta(t) E(t)-2 k_{2} E^{\prime}(t), \forall t \geq t_{0},
$$

which can be rewritten as

$$
\left(\vartheta(t) \mathcal{L}(t)+2 k_{2} E(t)\right)^{\prime}-\vartheta^{\prime}(t) \mathcal{L}(t) \leq-k_{1} \vartheta(t) E(t), \forall t \geq t_{0},
$$

using the fact that $\vartheta^{\prime}(t) \leq 0, \forall t \geq 0$, we have

$$
\left(\vartheta(t) \mathcal{L}(t)+2 k_{2} E(t)\right)^{\prime} \leq-k_{1} \vartheta(t) E(t), \forall t \geq t_{0} .
$$


By exploiting (2.29), we notice that

$$
\mathcal{R}(t)=\vartheta(t) \mathcal{L}(t)+2 k_{2} E(t) \sim E(t) .
$$

Consequently, for some positive constant $\lambda$, we obtain

$$
\mathcal{R}^{\prime}(t) \leq-\lambda \mathcal{R}(t) \vartheta(t), \forall t \geq t_{0}
$$

A simple integration of (2.32) over $\left(t_{0}, t\right)$ leads to

$$
\mathcal{R}(t) \leq \mathcal{R}\left(t_{0}\right) e^{-\lambda \int_{t_{0}}^{t} \vartheta(s) d s}, \forall t \geq t_{0} .
$$

Consequently, (2.24) is established by virtue of (2.29) and (2.33).

Remark 1. We give some examples to illustrate the energy decay rates obtained by Theorem 2.1. We consider the three different examples

$$
\text { If } g(t)=\beta_{1} e^{-\beta_{2} t} \text {, then } g^{\prime}(t)=-\vartheta(t) g(t) \text {, where } \vartheta(t)=\beta_{2} \text {, }
$$

then

$$
\begin{gathered}
E(t) \leq c_{0} e^{-\beta_{2} c_{1} t}, \forall t \geq 0, \\
\text { If } g(t)=\frac{\beta_{1}}{(1+t)^{\beta_{2}+1}}, \text { then } g^{\prime}(t)=-\vartheta(t) g(t) \text {, where } \vartheta(t)=\frac{\beta_{2}+1}{1+t},
\end{gathered}
$$

then

$$
E(t) \leq \frac{c_{0}}{(1+t)^{\left(\beta_{2}+1\right) c_{1}}}, \forall t \geq 0
$$

If $g(t)=\frac{\beta_{1}}{\left(e^{t\left(\frac{\pi}{2}-\text { arctgt }\right)} \sqrt{1+t^{2}}\right)^{\beta_{2}}}$, then $\quad g^{\prime}(t)=-\vartheta(t) g(t)$, where $\vartheta(t)=\beta_{2}\left(\frac{\pi}{2}-\operatorname{arctg} t\right)$, then

$$
E(t) \leq \frac{c_{0}}{c_{1}\left(e^{t\left(\frac{\pi}{2}-a r c t g t\right)} \sqrt{1+t^{2}}\right)^{\beta_{2}}}, \forall t \geq 0
$$

\section{References}

[1] A. Soufyane, M. Afilal, T. Aouam and M. Chacha. General decay of solutions of a linear one- dimensional porous-thermoelasticity system with a boundary control of memory type. Nonl. Anal., 72(11):3903-3910, 2010.

[2] T. A. Apalara. A general decay for a weakly nonlinearly damped porous system. J. Dyn. Control Syst., 25(3):311-322, 2019.

[3] T. A. Apalara. General decay of solution in one-dimensional porous-elastic system with memory. J. Math. Anal. Appl., 469(2):457-471, 2017. 
[4] T. A. Apalara. On the stabilization of a memory-type porous thermoelastic system. Bull. Malays. Math. Sci. Soc., 43:1433-1448(2020), https://doi.org/10.1007/s40840-019-00748-2.

[5] T. A. Apalara, C. A. Raposo and J. O. Ribeiro. Analyticity to transmission problem with delay in porous-elasticity. J. Math. Anal. Appl., 466(1):819-834, 2018.

[6] S. P. Yung, C. Q. Xu and L. K. Li. Stabilization of wave systems with input delay in the boundary control. ESAIM Control Optim. Calc. Var., 12(4):770-785, 2006.

[7] P. S. Casas and R. Quintanilla. Exponential decay in one-dimensional porousthermoelasticity. Mech. Res. Commun., 32(6):652-658, 2005.

[8] P. S. Casas and R. Quintanilla. Exponential stability in thermoelasticity with microtemperatures. Int. J. Eng. Sci., 43(1-2):33-47, 2005.

[9] S. C. Cowin and J. W. Nunziato. Linear elastic materials with voids. J. Elast., 13(2):125-147, 1983.

[10] S. C. Cowin and J. W. Nunziato. The viscoelastic behavior of linear elastic materials with voids. J. Elast., 15(2):185-191, 1985.

[11] R. Datko. Not all feedback stabilized hyperbolic systems are robust with respect to small time delays in their feedbacks. SIAM J. Control Optim., 26(3):697-713, 1988.

[12] E. H. Dill. Continum mechanics: elasticity, plasticity, viscoelasticity. CRC Press,TaylorFrancis Group, New York, page pp 368, 2006.

[13] A Fareh and S. A. Messaoud. Energy decay for a porous thermoelastic system with thermoelasticity of second sound and with a non-necessary positive definite energy. Appl. Math. Comput., 293(15):493-507, 2017.

[14] M. A. Goodman and S. C. Cowin. A continuum theory for granular materials. Arch. Ration. Mech. Anal., 44(4):249-266, 1972.

[15] A. Magana and R. Quintanilla. On the time decay of solutions in one-dimensional theories of porous materials. Int.J.Solids Struct., 43(11-12):3414-3427, 2006.

[16] A. S. Nicaise and C. Pignotti. Stabilization of the wave equation with boundary or internal distributed delay. Diff. Int. Equs., 21(9-10):935-958, 2008.

[17] J. W. Nunziato and S. C. Cowin. A nonlinear theory of elastic materials with voids. Arch. Ration. Mech. Anal., 72(2):175-201, 1979. 
[18] D. Ouchenane. A stability result of a timoshenko system in thermoelasticity of second sound with a delay term in the internal feedback. Georgian Math. J., 21(4):475-489, 2014.

[19] J. E. M. Rivera, P. X. Pamplona and R. Quintanilla. Stabilization in elastic solids with voids. J. Math. Anal. Appl., 350(1):37-49, 2009.

[20] J. E. M. Rivera, P. X. Pamplona and R. Quintanilla. On the decay of solutions for porouselastic system with history. J. Math. Anal. Appl., 379(2):682-705, 2011.

[21] R. Quintanilla. Slow decay in one-dimensional porous dissipation elasticity. Appl. Math. Lett., 16(4):487-491, 2003.

[22] J. Lagnese, R. Datko and M. P. Polis. An example on the effect of time delays in boundary feedback stabilization of wave equations. SIAM J. Control Optim., 24(1):152-156, 1986.

[23] J. E. M. Rivera and R. Quintanilla. On the time polynomial decay in elastic solids with voids. J. Math. Anal. Appl., 338(2):1296-1309, 2008.

[24] M. L. Santos and D. A. Junior. On porous-elastic system with localized damping. Z. Angew. Math. Phys., 67(63):1-18, 2016.

[25] A. Soufyane. Energy decay for porous-thermo-elasticity systems of memory type. Appl. Anal., 87(4):451-464, 2008.

[26] I. H. Suh and Z. Bien. Use of time delay action in the controller design. IEEE Trans. Automat. Control., 25(3):600-603, 1980.

[27] S. Zheng and Z. Liu. Semigroups associated with dissipative systems. Chapman Hall/CRC: Boca, Raton, page pp 224, 1999.

Abdelbaki Choucha Department of Mathematics, Faculty of Exact Sciences, University of El Oued, B.P. 789, El Oued 39000, Algeria

E-mail: abdelbaki.choucha@gmail.com

Djamel Ouchenane Laboratory of pure and applied Mathematics, Amar Teledji Laghouat University, Algeria

E-mail: ouchenanedjamel@gmail.com

Khaled Zennir Department of Mathematics, College of Sciences and Arts, Al-Ras, Qassim University, Kingdom of Saudi Arabia

E-mail: k.Zennir@qu.edu.sa 